\title{
COMPOSIÇÃO E DISTRIBUIÇÃO DA FAMÍLIA PARACALANIDAE (COPEPODA: CALANOIDA) AO LARGO DE SÃO SEBASTIÃO, ESTADO DE SÃO PAULO-BRASIL, COM ÊNFASE EM TRÊS ESPÉCIES DE Paracalanus
}

\author{
Luz Amelia Vega-Pérez \& Simone Hernandez \\ Instituto Oceanográfico da Universidade de São Paulo \\ (Caixa Postal 66149,05315-970 São Paulo, SP Brasil)
}

- Abstract: The qualitative structure as well as the distribution of Paracalanidae species off São Sebastião region, São Paulo State, Brazil, were studied from samples taken during Summer 1994 at 43 stations. Seven species belonging to the genera Paracalanus and Acrocalanus were identified: Paracalanus quasimodo, Paracalanus indicus, Paracalanus aculeatus, Paracalanus campaneri, Paracalanus crassirostris, Paracalanus nanus and Acrocalanus longicornis. The three first species were the most frequent and abundant in the area. The populations of Paracalanidae were composed basically of adult females, males and copedids IV-V and their densities were higher in the region localized between $20 \mathrm{~m}$ and $74 \mathrm{~m}$. depth. The vertical distribution also was studied for a $24-\mathrm{h}$ period at a fixed station (46 m. depth). High densities of Paracalanidae were found mostly above the thermocline specially during the nocturnal period, indicating a nocturnal upward migration.

- Resumo: A composição e distribuição das espécies da família Paracalanidae ao largo de São Sebastião, Estado de São Paulo, Brasil, foram estudadas a partir de amostras coletadas no verão de 1994, em 43 estações. Sete espécies pertencentes aos gêneros Paracalanus e Acrocalanus foram identificadas: Paracalanus quasimodo, Paracalanus indicus, Paracalanus aculeatus, Paracalanus campaneri, Paracalanus crassirostris, Paracalanus nanus e Acrocalanus longicornis, sendo as três primeiras espécies as mais freqüentes e numerosas. As populações de Paracalanidae foram formadas por fêmeas e machos adultos, seguidos dos estágios de copepóditos IV e V, sendo os maiores valores de densidade detectados na região localizada entre as isóbatas dos $20 \mathrm{~m}$ e $74 \mathrm{~m}$. A distribuição vertical dos Paracalanidae foi estudada em uma estação fixa, de 24 horas de duração, onde a profundidade atingia $46 \mathrm{~m}$. As maiores densidades foram encontradas acima da termoclina, principalmente durante o período noturno, indicando que essas espécies se deslocam para as camadas superiores durante a noite.

- Descriptors: Copepod distribution, Paracalanidae, Population density, South Atlantic, São Sebastião: SP, Brazil.

- Descritores: Distribuição de copépodos, Paracalanidae, Densidade da população, Atlântico Sul, São Sebastião: SP, Brasil. 


\section{Introdução}

Os Copepoda são os organismos dominantes na biomassa mesozooplanctônica das regiões oceânicas, cuja distribuição e abundância estão intimamente relacionadas com a estrutura hidrológica local (Seguin et al., 1994). Recentemente, eles vem sendo considerados como os produtores secundários mais importantes do ecossistema pelágico marinho, tanto em termos de abundância como de biomassa (Hopcroft \& Roff, 1996).

A ordem Calanoida é constituída de 40 famílias (Damkaer, 1996) cujos componentes são em sua grande maioria holoplanctônicos (Rippingale, 1994), herbívoros e onívoros (Kouwenberg, 1994). Como segundo nível da cadeia trófica marinha, eles servem de alimento para as espécies zooplanctófagas (Nakata, 1988, Hillgruber et al., 1995; Pedreira, 1997) e são de grande importância na produção de materia orgânica (Paffenhöfer \& Knowles, 1979; Angel, 1984; Gonzáles et al., 1994), bem como no consumo e degradação das pelotas fecais produzidas nas camadas de superfície (Smetacék, 1980; Sasaki et al., 1988).

Os copépodos calanóides pertencentes à família Paracalanidae possuem ampla distribuição geográfica, sendo freqüentemente encontrados nas regiões tropicais e subtropicais (Bowman, 1971), onde cumprem papel preponderante na trofodinâmica marinha, já que podem atuar como reguladores do fitoplâncton através da atividade de "grazing" (Turner, 1994). Nas águas brasileiras foram identificadas até o momento dez espécies (Björnberg, 1981), das quais sete são comumente encontradas no litoral do Estado de São Paulo (Vega-Pérez, 1993).

O presente trabalho tem como objetivo estudar a distribuição de 3 espécies da familia Paracalanidae que ocorrem na região de São Sebastião, Estado de São Paulo, baseada em dados obtidos de amostras coletadas no verão de 1994 .

\section{Material e métodos}

\section{Coleta e obtenção das amostras}

Amostras de zooplâncton foram obtidas em São Sebastião, litoral norte do Estado de São Paulo (Lat. $23^{\circ} 35^{\prime}$, Long. $45^{\circ} 47^{\prime}$ ), entre 7 e 10 de fevereiro de 1994, com o auxílio do N/Oc. "Prof. W. Besnard", dentro do Projeto Integrado "Oceanografia da Plataforma Interna de São Sebastião - OPISS". A área estudada (Fig. 1) foi dividida em 5 subáreas por Katsuragawa (1995):

- Área 1 , interna à isóbata de $20 \mathrm{~m}$, localizada ao sul da ilha de São Sebastião;
- Área 2, interna à isóbata de $20 \mathrm{~m}$, localizada ao norte da ilha de São Sebastião;

- Área 3, localizada entre as isóbatas de $20 \mathrm{~m} \mathrm{e}$ $50 \mathrm{~m}$, ao sul da ilha de São Sebastião;

- Área 4, localizada entre as isóbatas de $20 \mathrm{~m} \mathrm{e}$ $50 \mathrm{~m}$, ao norte da ilha de São Sebastião;

- Área 5, externa à isóbata dos $50 \mathrm{~m}$.

Nessas áreas, um total de 43 estaç̃os hidrográficas foram amostradas com rede Bongô dupla de $60 \mathrm{~cm}$ de diâmetro de boca, malhas de $0,333 \mathrm{~mm}$ (rede fina) e $0,505 \mathrm{~mm}$ (rede regular), munidas de fluxômetro. Os arrastos oblíquos foram feitos às profundidades mínima de $9 \mathrm{~m}$ e máxima de $70 \mathrm{~m}$. Neste trabalho, utilizaram-se apenas as amostras coletadas com a rede fina.

A distribuição vertical dos membros da família Paracalanidae foi analisada a partir de amostras coletadas durante 24 horas consecutivas $(6 \mathrm{~h} ; 12 \mathrm{~h}$; $18 \mathrm{~h} ; 24 \mathrm{~h}$ e $6 \mathrm{~h}$ ), entre os dias 26 e 27 de fevereiro de 1994, numa estação fixa de $46 \mathrm{~m}$ de profundidade localizada ao sul da ilha de São Sebastião (Fig. 1). Os arrastos verticais foram feitos com rede de fechamento, de $0,5 \mathrm{~m}$ de diâmetro de boca e malha de $0,200 \mathrm{~mm}$, acima da termoclina em profundidades máximas de $14-0 \mathrm{~m}$ e mínimas de 5-0 $\mathrm{m}$. Abaixo da termoclina, esses máximos e mínimos foram de 44$38 \mathrm{~m}$ e 44-28 m, respectivamente. $O$ volume de água filtrada foi estimado seguindo-se a fórmula $\mathrm{V}=\pi . \mathrm{r}^{2} . \mathrm{z}$ (Omori \& lkeda, 1992).

Dados de temperatura e salinidade foram obtidos em cada uma das estações. Logo após as coletas, todas as amostras foram fixadas em formol $4 \%$ neutralizado.

\section{Tratamento das amostras}

O volume de cada amostra foi calculado através do método de Kramer et al., (1972). Na determinação da volumetria não foram considerados os organismos gelatinosos tais como salpas, medusas e quetógnatos, o mesmo acontecendo com os ovos e larvas de peixes.

$\mathrm{Na}$ triagem e separação dos Paracalanidae utilizaram-se pinças de joalheiro, pipetas Pasteur e estereomicroscópio Wild M7. A identificação das espécies foi feita com base em Bowman (1971), Lawson \& Grice (1973) e Björnberg (1981).

As associações faunísticas foram analisadas utilizando-se os índices de similaridade de Morisita modificado por Horn (1966), para o modo Q, e o de dissimilaridade de Bray-Curtis para o modo $\mathrm{R}$ (Legendre \& Legendre, 1983), este calculado com os dados de densidade das 7 espécies de Paracalanidae transformados por $\log (x+1)$. A análise de agrupamento foi realizada através do método da média aritmética ponderada (UPGMA), sendo representada na forma de dendrograma. 


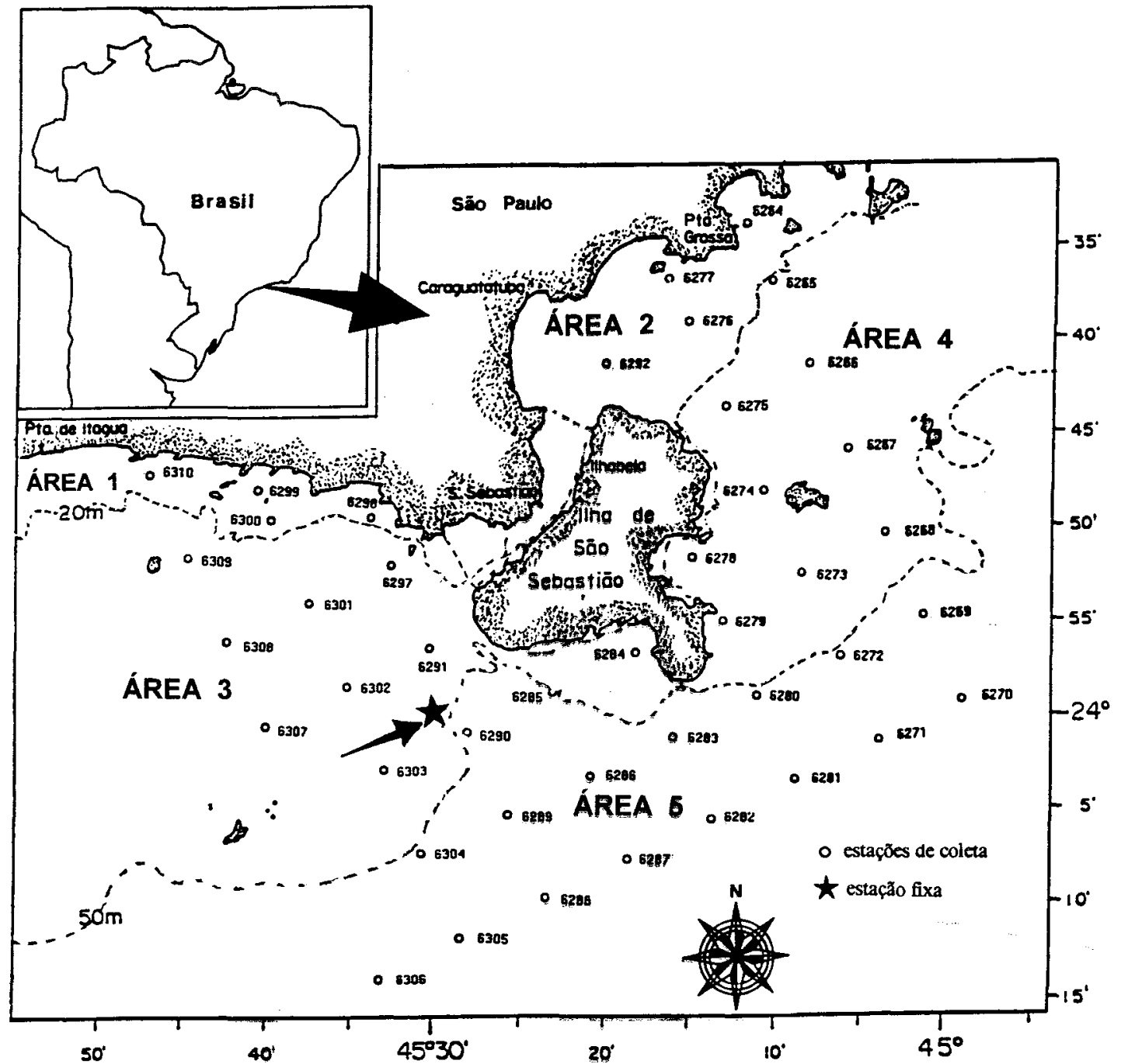

Fig. 1. Mapa da região de São Sebastião, dividida em 5 subáreas, mostrạndo a localização das estações de coleta (0) e a estação fixa ( $\star$, no verão de 1994.

\section{Resultados}

A área estudada, no verão de 1994 , esteve sob o domínio da Água Costeira (AC), caracterizada por apresentar temperaturas altas que variam de $21,0^{\circ}$ a $29,0^{\circ} \mathrm{C}$ e salinidades de 31 a 35,5 .

A Água Central do Atlântico Sul (ACAS), com temperaturas abaixo dos $20,0^{\circ} \mathrm{C}$ e salinidades variando entre 35 e 36 , esteve presente nas áreas 3,4 e 5 , nas camadas compreendidas entre 20 e $68 \mathrm{~m}$ de profundidade.

$\mathrm{Na}$ região de São Sebastião, a família Paracalanidae foi formada pelos gêneros Paracalanus e Acrocalanus. $O$ gênero Paracalanus apresentou 6 espécies: Paracalanus quasimodo Bowman, 1971; Paracalanus indicus Woffenden, 1905; Paracalanus aculeatus Giesbrecht, 1888; Paracalanus campaneri Bjornberg, 1979; Paracalanus crassirostris F. Dahl, 1894 e Paracalanus nanus G. O. Sars, 1907. O gênero
Acrocalanus esteve representado por apenas uma espécie, Acrocalanus longicornis Giesbrecht, 1888.

A distribuição e o número de indivíduos por $\mathrm{m}^{2}$ dos membros da família Paracalanidae, em cada estaç de de coleta, são apresentados na Figura 2. Dentre as espécies identificadas, $P$. quasimodo foi a mais freqüente e numerosa, seguida por $P$. indicus, $P$. aculeatus e $P$. campaneri. Em contraposição, $P$. nanus, $P$. crassirostris e $A$. longicornis ocorreram apenas em algumas estações e em quantidades reduzidas.

Paracalanus quasimodo ocorreu em quase toda a área estudada, isto é, em 39 das 43 estações (Fig. 3a). Na população houve predomínio de fêmeas tanto adultas como no estágio de copepódito $\mathrm{V}$. Indivíduos machos adultos e no estágio de copepódito $\mathrm{V}$, bem como o estágio de copepódito IV, de ambos os sexos, ocorreram em baixas densidades, o mesmo acontecendo com os copepóditos IIII. 


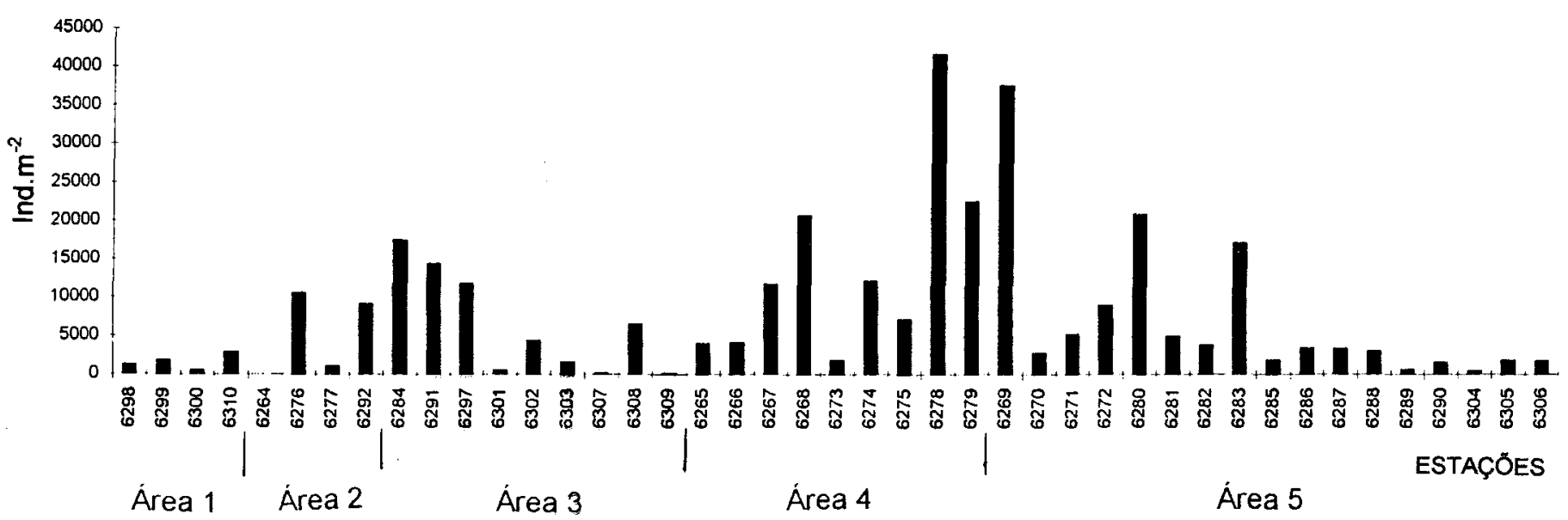

Fig. 2. Variação espacial do número de indivíduos por $\mathrm{m}^{2}$ da família Paracalanidae, na região de São Sebastião, no verão de 1994.

Os maiores valores de densidade da espécie foram observados nas estações St. 6269 (26393 ind. $\left.\mathrm{m}^{-2}\right)$ e St. 6278 (21899 ind. $\left.\mathrm{m}^{-2}\right)$, localizadas nas áreas 5 e 4, respectivamente. Valores mínimos foram verificados nas estações St. 6264 (3 ind. $\left.\mathrm{m}^{-2}\right)$ e St. 6309 (46 ind. $\mathrm{m}^{-2}$ ), situadas nas áreas 2 e 3 , respectivamente. $P$. quasimodo esteve ausente $\mathrm{em}$ quatro estações localizadas ao sul da Itha de São Sebastião.

Paracalanus indicus ocorreu em 28 das 43 estações amostradas (Fig. 3b). A estrutura da população desta espécie foi semelhante à de $P$. quasimodo, já que houve predomínio de fêmeas adultas e de copepódito $\mathrm{V}$ fềmea. Os copepóditos IV fêmea e V macho, bem como os machos adultos ocorreram em quantidades mínimas, o mesmo acontecendo com o copepódito III. Os maiores valores de densidade, 19956 ind. $^{-2}$ (St. 6278) e 8137 ind. $\mathrm{m}^{-2}$ (St. 6279), foram registrados na área 4, enquanto que os menores valores, 15 ind. ${ }^{-2}$ (St. 6309) e 56 ind.m $\mathrm{m}^{-2}$ (St. 6299), foram verificados nas áreas $1 \mathrm{e} 3$, respectivamente.

Paracalanus aculeatus foi encontrado em 40 das 43 estações de coleta (Fig. 3c). Sua população constituíu-se basicamente de fêmeas e machos adultos. Os copepóditos V e IV, de ambos os sexos, ocorreram em pequena quantidade. Os maiores valores de densidade para esta espécie foram encontrados nas estações St. 6291 (6927 ind. $\mathrm{m}^{-2}$ ) e St.6268 (6071 ind. $\mathrm{m}^{-2}$ ), localizadas nas áreas 3 e 4, enquanto que os menores valores foram observados na área 1, St. $6300\left(80\right.$ ind. $\left.\mathrm{m}^{-2}\right)$ e na área 3, St. 6309 (15 ind. $\mathrm{m}^{-2}$ ).

A análise de agrupamento (Fig. 4) distinguiu 3 grupos de estações (A, $\mathrm{B}$ e $\mathrm{C}$ ) em função das semelhanças quanto à composição específica em nivel de mais de $65 \%$ de similaridade. O grupo $A$ foi formado por 29 estações, das quais 16 estiveram sob a influência da ACAS a partir dos $65 \mathrm{~m}$ de profundidade. A espécie dominante foi $P$. quasimodo seguida por $P$. aculeatus e $P$. indicus. O grupo B foi constituído por 9 estações, delas 6 localizadas entre as isóbatas dos $12 \mathrm{~m}$ e $40 \mathrm{~m}$ e, portanto, dominadas totalmente pela AC. As outras 3 estações, por estarem localizadas em áreas de maior profundidade, tiveram influência da ACAS a partir dos $60 \mathrm{~m}$ de profundidade. As espécies dominantes foram $P$. quasimodo e $P$. aculeatus. $\mathrm{O}$ grupo $\mathrm{C}$ reuniu 5 estações, 4 delas localizadas além da isóbata dos 50 $\mathrm{m}$, onde a ACAS esteve presente a partir dos $50 \mathrm{~m}$. Neste grupo, $P$. aculeatus e $P$. quasimodo foram as espécies dominantes.

A análise inversa (Fig. 5) evidenciou um grupo de espécies, Grupo 1, formado $P$. quasimodo, $P$. indicus e pela associação de $P$. aculeatus e $P$. campaneri, sendo que esta última espécie ocorreu em baixa freqüência. As outras 3 espécies incluídas na análise, Grupos 2, 3 e 4, mostraram-se independentes.

Com relação à distribuição vertical dos membros da família Paracalanidae (Fig. 6), verificou-se que eles ocorreram em maior densidade $\left(\mathrm{n}^{0}\right.$.ind. $\left.\mathrm{m}^{-3}\right)$ acima da termoclina, durante o período noturno, principalmente às $24 \mathrm{~h}$.

$P$. quasimodo ocorreu em maior quantidade acima da termoclina, especialmente às $24 \mathrm{~h}$. Abaixo da termoclina, a densidade foi em geral menor. Contudo, um pequeno aumento nos valores de densidade foi detectado por volta das $6 \mathrm{~h}$ da manhã (Fig. 7a). De um modo geral houve predomínio de fêmeas adultas e copepódito $V$ fêmea acima e abaixo da termoclina. Os copepóditos V macho, IV fềmea $\mathrm{e}$ macho, assim como o copepódito III ocorreram em maior número abaixo da termoclina, principalmente às $18 \mathrm{~h}$ (Figs 7b-c). 


\section{A}
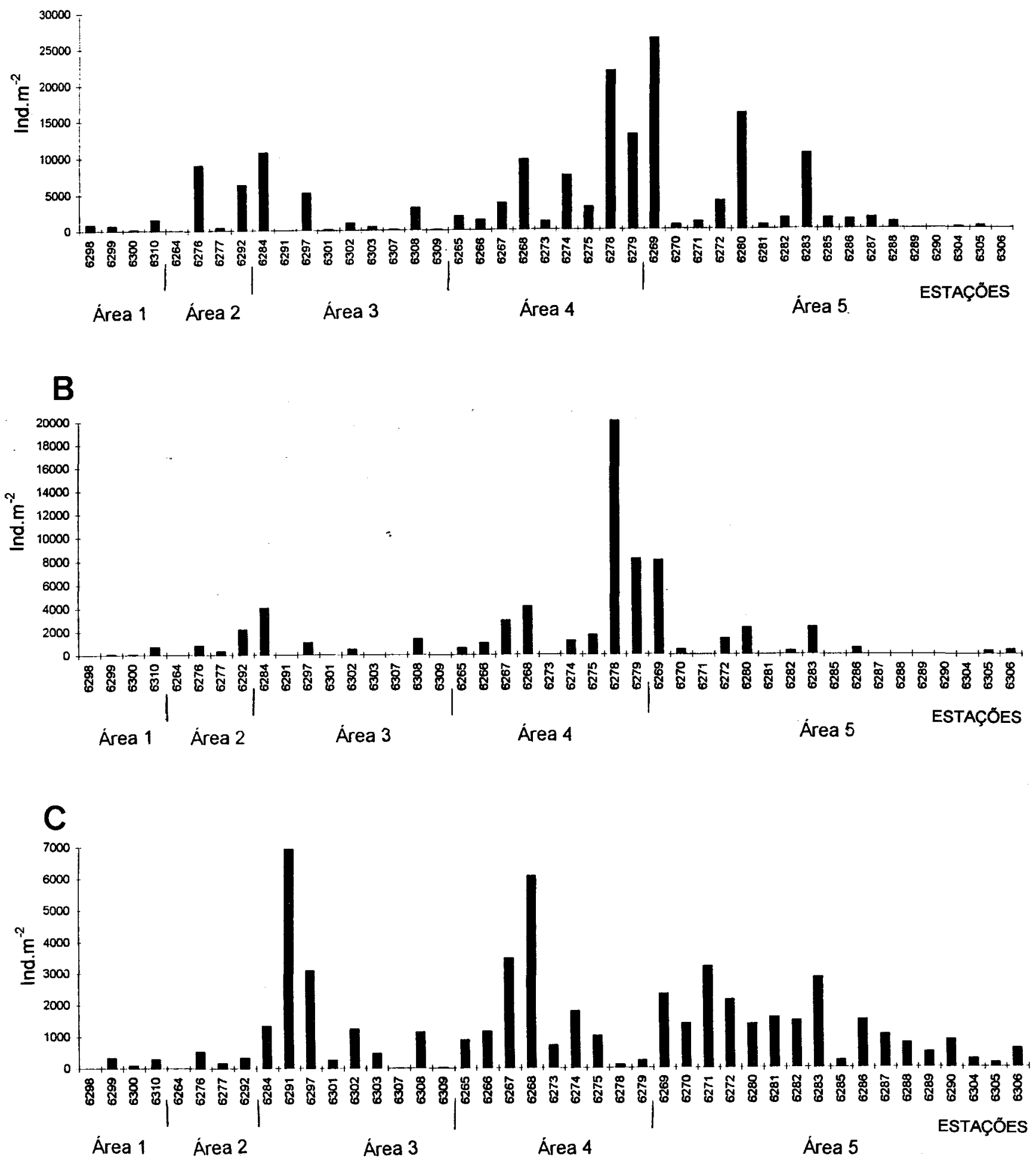

Fig. 3. A. Variação espacial do número de indivíduos por $\mathrm{m}^{2}$ da espécie Paracalanus quasimodo, na região de São Sebastião, no verão de 1994. B. Variação espacial do número de indivíduos por $\mathrm{m}^{2}$ da espécie Paracalanus indicus, na região de São Sebastião, no verão de 1994. C. Variação espacial do número de indivíduos por $\mathrm{m}^{2}$ da espécie Paracalanus aculeatus, na região de São Sebastião, no verão de 1994. 


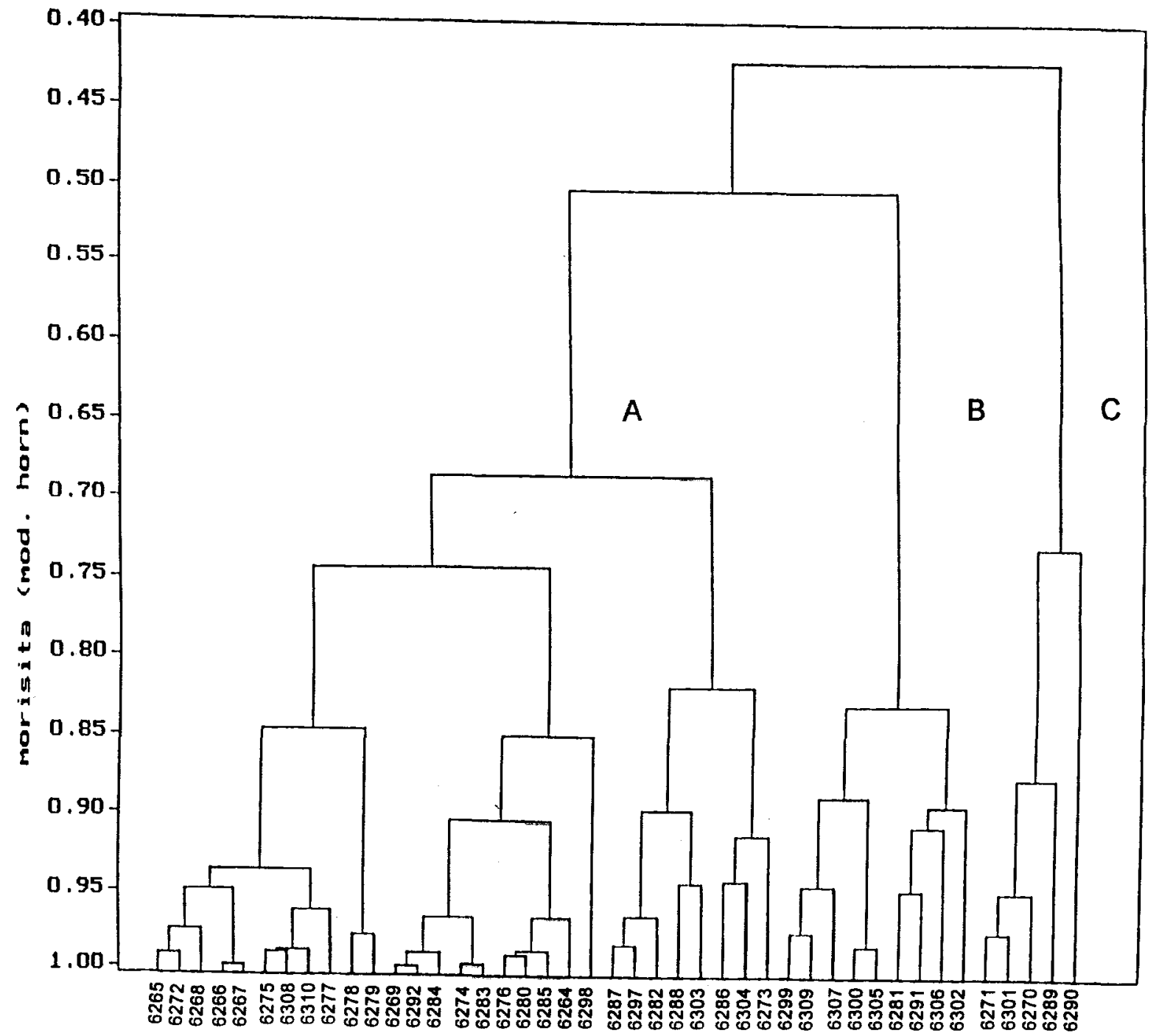

Fig. 4. Dendrograma da análise de agrupamento das 43 estações de coleta, no verão de 1994. Índice de Similaridade de Morisita (Horn modificado) e agrupamento por UPGMA. Os três "clusters" diferenciados são marcados com as letras A - C.

A distribuição vertical de $P$. indicus foi muito semelhante à de $P$. quasimodo. $\mathrm{O}$ maior número de indivíduos por $\mathrm{m}^{3}$ foi encontrado acima da termoclina no período compreendido entre 18 e $24 \mathrm{~h}$. Abaixo da termoclina foi observada uma maior concentração de organismos apenas às $6 \mathrm{~h}$ (Fig. 8a). Com relação à estrutura da população, houve um predomínio de fềmeas adultas e do estágio de copepódito IV macho acima da termoclina, principalmente às $24 \mathrm{~h}$. Abaixo da termoclina, foi encontrado maior número de fêmeas jovens, copepóditos $\mathrm{V}$ e IV, no horário das $6 \mathrm{~h}$ do dia 27 de fevereiro (Figs 8b-c).
O pico máximo de ocorrência de $P$. aculeatus foi observado acima da termoclina, principalmente às $24 \mathrm{~h}$. Abaixo da termoclina, a maior densidade foi encontrada às $6 \mathrm{~h}$ do segundo dia de coleta, superando os valores de densidade encontrados acima da termoclina (Fig. 9a). A população foi constituída basicamente de fêmeas adultas e copepódito $\mathrm{V}$ macho, sendo os máximos valores detetados às $24 \mathrm{~h}$, acima da termoclina. Abaixo da termoclina, o copepódito $\mathrm{V}$ macho $\mathrm{e}$ as fêmeas adultas foram mais abundantes às $18 \mathrm{~h}$ e $6 \mathrm{~h}$, respectivamente (Figs 9b-c). 


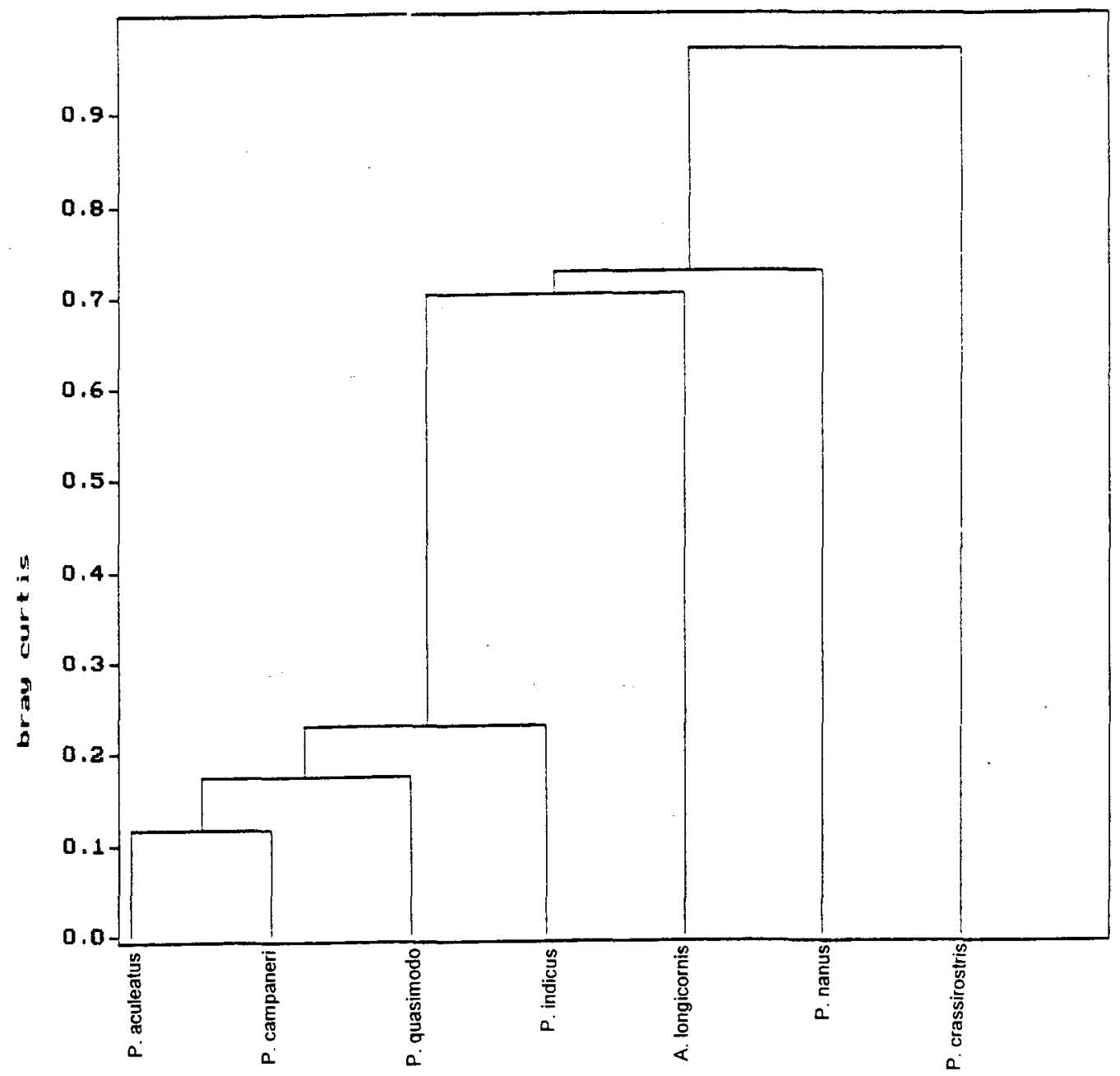

Fig. 5. Dendrograma da análise de agrupamento das espécies, no verão de 1994. Indice de Similaridade de Morisita (Horn modificado). Índice de dissimilaridade de Bray-Curtis e agrupamento por UPGMA.

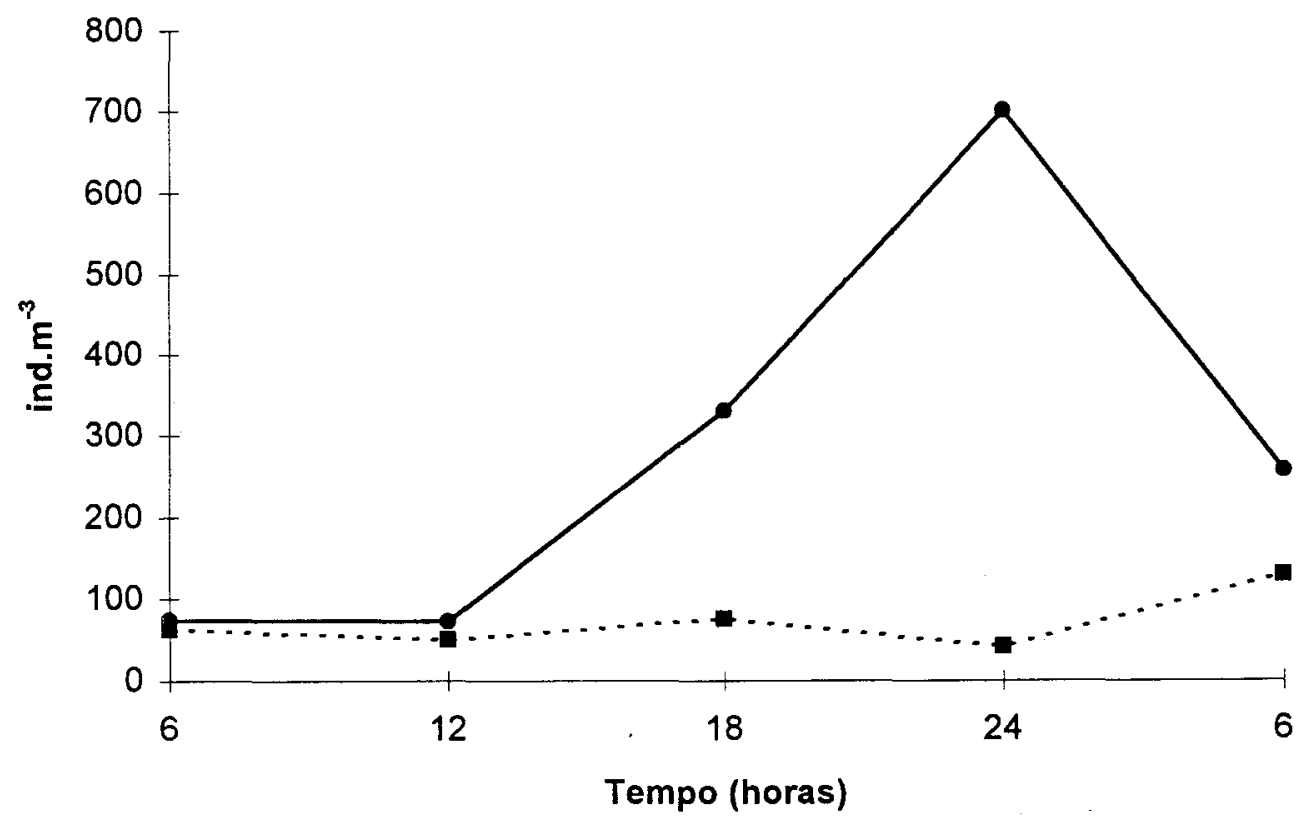

Fig. 6. Distribuição vertical e variação temporal dos espécimes da família Paracalanidae, acima $(\longrightarrow-$ ) e abaixo ( …-...-) da termoclina, na região de São Sebastião, no verão de 1994. 
A

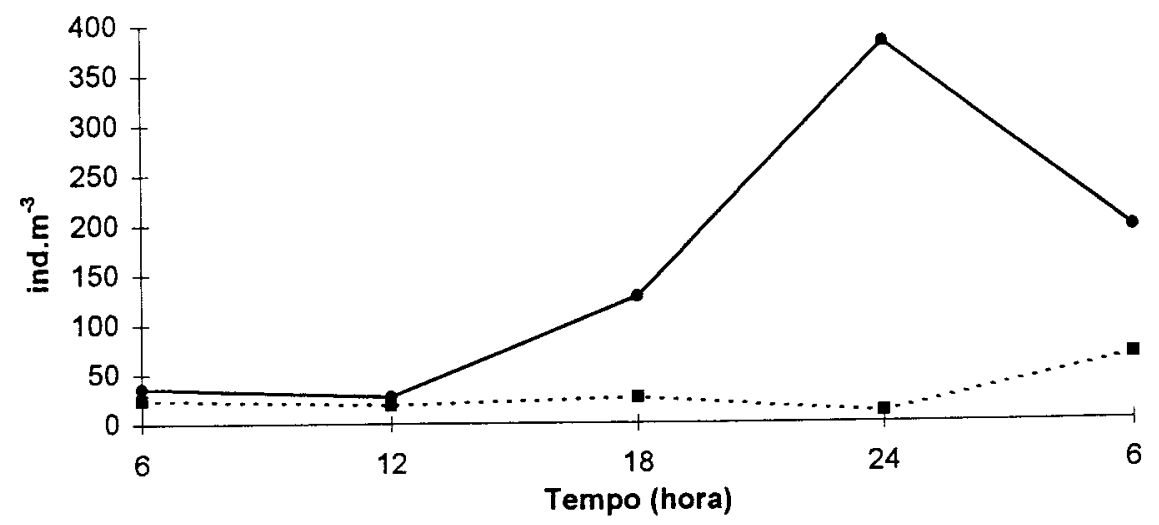

B

ECIII

amac.IV

mmac. $V$

mac. adulto

afem. IV

afem. $V$

- fem. adulta

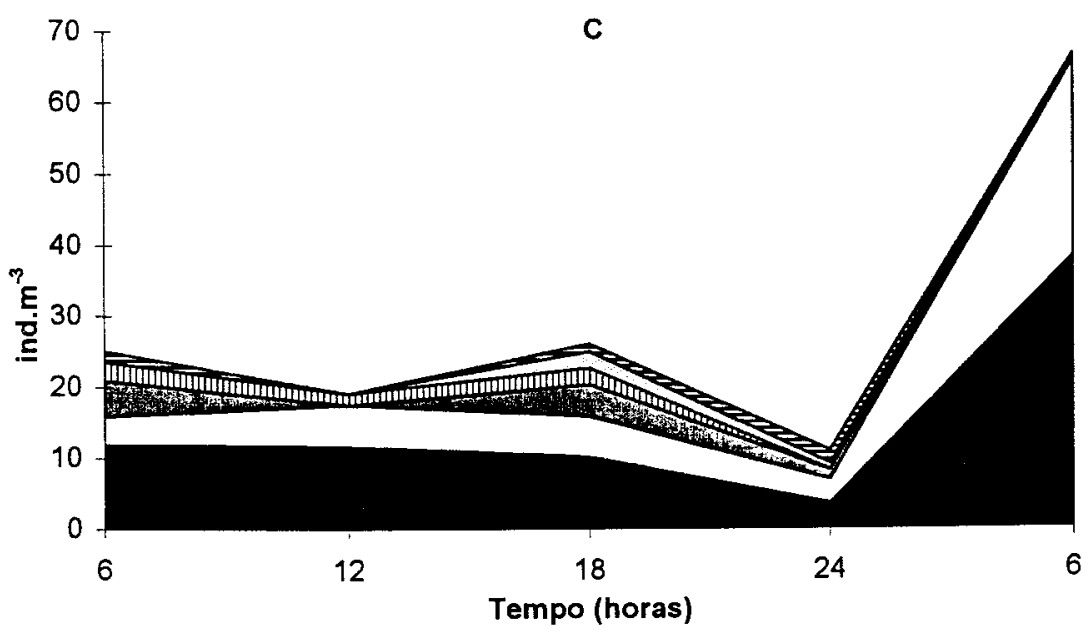

Fig. 7. Distribuição vertical e variação temporal de Paracalanus quasimodo: (A) acima (- - ) e abaixo ( $-\cdots-\cdots$ termoclina e dos diferentes estágios de desenvolvimento acima (B) e abaixo da termoclina (C). 
A
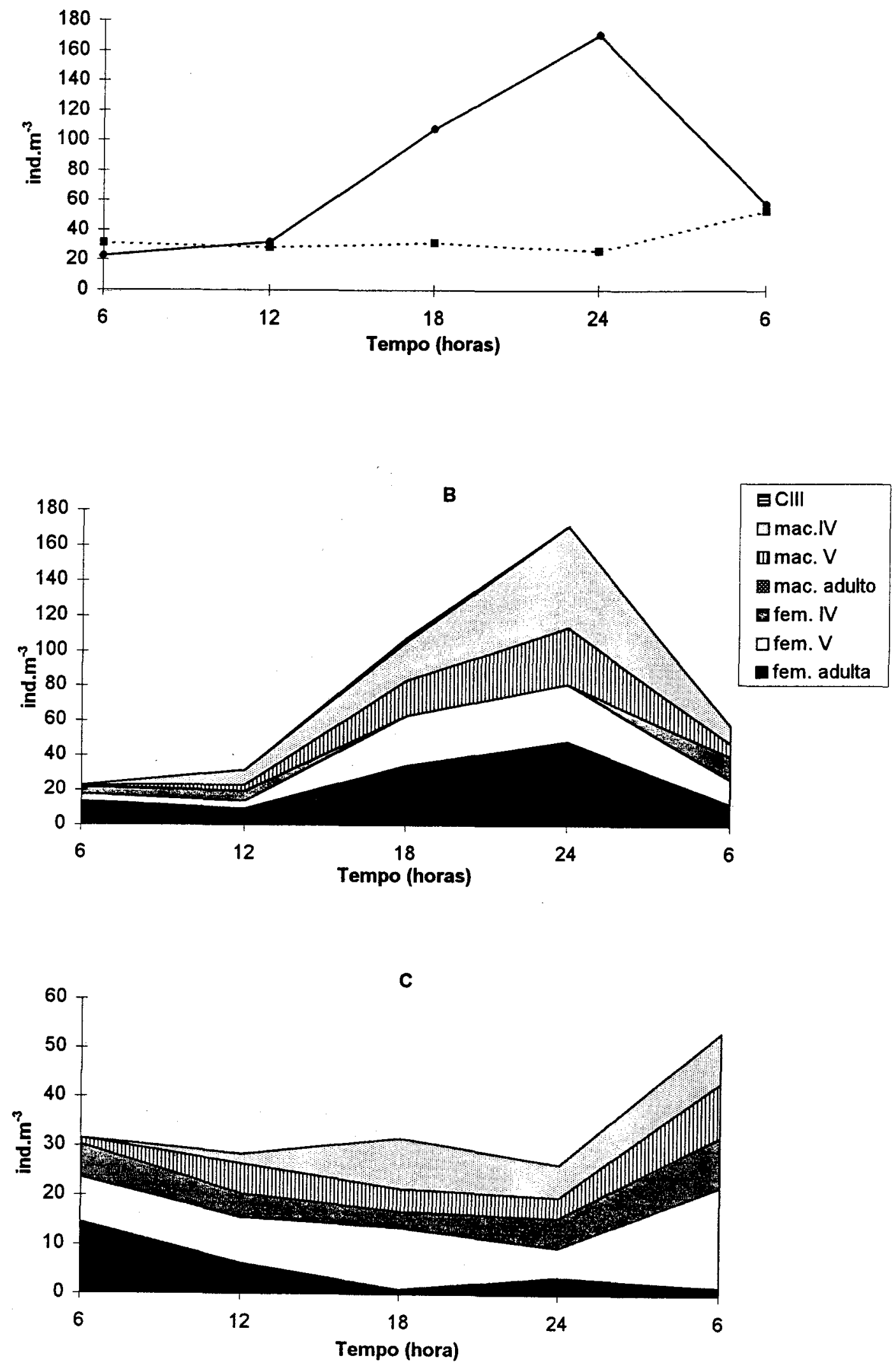

Fig. 8. Distribuição vertical e variação temporal da espécie Paracalanus indicus: (A) acima (- - ) e abaixo ( - - - ) da termoclina e dos diferentes estágios de desenvolvimento acima (B) e abaixo da termoclina (C). 
A

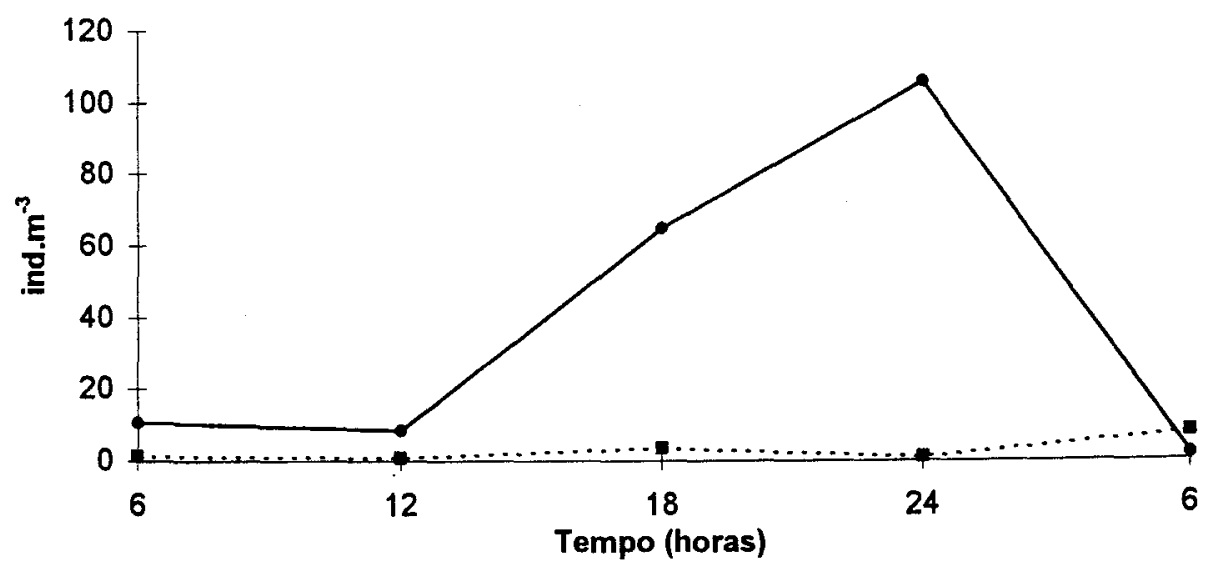

B $\quad$ 目CIII
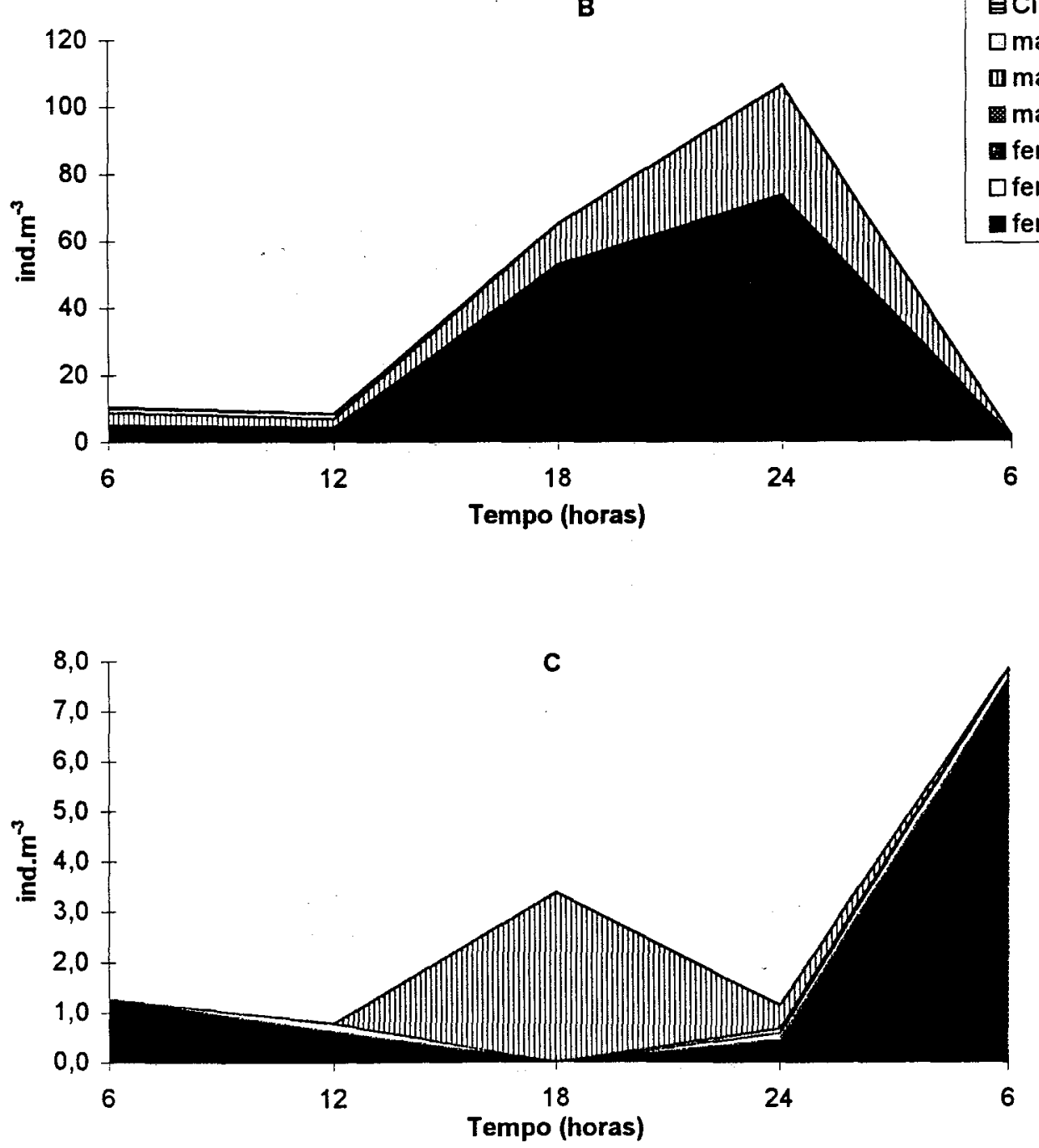

Fig. 9. Distribuição vertical e variação temporal da espécie Paracalanus aculeatus: (A) acima ( $\longrightarrow-$ ) e abaixo ( - - - $-\cdots$ da termoclina e dos diferentes estágios de desenvolvimento acima (B) e abaixo da termoclina (C). 


\section{Discussão}

A composição da família Paracalanidae em São Sebastião foi similar à relatada para a costa brasileira (Björnberg, 1981) e região de Ubatuba (Vega-Pérez, 1993), porém o número de espécies desta família encontrado por Gaeta et al. (1990) no Canal de São Sebastião foi menor. Das 3 espécies de Paracalanus identificadas por estes últimos autores, $P$. parvus, $P$. quasimodo e $P$. crassirostris, somente a primeira espécie esteve ausente nas amostras estudadas.

No ecossistema marinho os processos físicos e biológicos podem afetar a composição, abundância e distribuição dos organismos planctônicos (Richerson et al., 1978; Coyle et al., 1990; Grant, 1991; Mann, 1991). Dentre estes a temperatura (Razouls \& Thiriot, 1968), salinidade, bem como a quantidade e qualidade do alimento têm papel preponderante (Raymont, 1983; Nielsen \& Sabatini, 1996).

A ocorrência e distribuição dos copépodos em uma determinada área depende das condições hidrobiológicas (Seguin et al., 1994). As espécies da família Paracalanidae são freqüentemente encontradas nas águas neríticas e oceânicas das regiões tropicais e subtropicais com temperaturas e salinidades altas (Björnberg, 1963; 1981). A ocorrência dos Paracalanidae em toda a área estudada, no verão de 1994, sugere que eles estiveram associados à Água Costeira (AC). Esta massa de água, que dominou toda a área, caracterizou-se por apresentar temperaturas de até $29^{\circ} \mathrm{C}$ e salinidade de até 35,5 .

De acordo com Björnberg (1980), P. quasimodo é a espécie de Paracalanus mais freqüente $\mathrm{e}$ abundante em águas costeiras quentes de salinidade de 30 e de água de plataforma quente. Segundo esse mesmo autor, $P$. indicus também é típico dessas regiões, porém menos freqüente e abundante que o precedente. Dentre os Paracalanus que ocorrem na região de São Sebastião, $P$. quasimodo foi a espécie dominante seguida de $P$. indicus, confirmando assim os relatos de Björnberg (op. cit.).

A espécie $P$. aculeatus é típica de águas quentes de plataforma e oceânicas, com salinidade acima de 36 e temperaturas maiores do que $20^{\circ} \mathrm{C}$ (Björnberg, $o p$. cit.). Em São Sebastião essa espécie foi a terceira em ordem de importância, sendo freqüente nas estações com profundidades maiores e onde a ACAS esteve presente a partir dos $25 \mathrm{~m}$.

Valdes et al. (1990) verificaram que não houve diferenças significativas na abundância e biomassa de pequenas espécies de copépodos, especialmente Paracalanus, coletadas nas estações localizadas próximo da costa e nas mais afastadas da região da Galicia (Espanha). Os maiores valores de densidade das 3 espécies de Paracalanus estudadas foram encontrados nas estações localizadas além da isóbata dos $50 \mathrm{~m}$, nas áreas 4 e 5 , o que pode ser atribuído à presença da ACAS nas camadas compreendidas entre os 25 e $68 \mathrm{~m}$ de profundidade. Segundo Aidar et al. (1993), a ACAS é uma massa de água que está associada a altos teores de nutrientes e produção de fitoplâncton. Esta característica teria propiciado o desenvolvimento dos Paracalanus nas áreas 4 e 5 (Fig. 2), visto que suas espécies são em sua grande maioria herbívoras (Björnberg \& Wilbur, 1968; Kouwenberg, 1994). Em contraposição, nas áreas $1 \mathrm{e}$ 2 , as populações de $P$. quasimodo, $P$. indicus e $P$. aculeatus apresentaram os menores valores de densidade. Essas áreas, localizadas entre o litoral e a isóbata dos $20 \mathrm{~m}$, estiveram sob o domínio total da AC que, apesar dos seus moderados valores de clorofila a e nutrientes (Gianesella-Galvão, 1996), parece não ter exercido influência direta no desenvolvimento dessas três espécies durante o período de estudo.

A turbidez e turbulência são os fatores que mais aumentam em direção a costa. Para Eisma \& Kalf (1987) a quantidade de material em suspensão aumenta drásticamente nas regiões costeiras. Mudanças no tamanho, quantidade e qualidade da matéria orgânica particulada aliada à turbulência, em pequena escala, podem afetar indiretamente a atividade dos copepóditos (Kiørboe et al., 1990; Saiz \& Alcaraz, 1991), especialmente no que diz respeito à capacidade de capturar e manipular o seu alimento (Soetaert \& Herman, 1994). Ao que tudo indica fatores como o material em suspensão, presente em grandes quantidades na área $1 \mathrm{e}$ em quantidades moderadas na área 2 (Gianesella-Galvão, op. cit.), estariam afetando a composição, abundância e distribuição dos Paracalanus ao impedir ou dificultar o processo de alimentação.

Os valores médios de densidade encontrados em algumas estaç̃̃es localizadas na área 3 , situada entre a isobata dos 20 e $50 \mathrm{~m}$, podem ser atribuídos à presença da ACAS nas camadas mais profundas. Raymont (1983) reportou a ocorrência de espécimes adultos e jovens de $P$. aculeatus no Canal da Mancha durante $o$ ano inteiro, especialmente no período compreendido entre fevereiro e agosto. Resultados semelhantes foram observados no presente trabalho, já que as populações de $P$. quasimodo, $P$. indicus e $P$. aculeatus também estiveram constituídas basicamente de fềmeas adultas, bem como de copepóditos V e IV.

Autores como Katsuragawa (1985), apontaram vários problemas relacionados à eficiência dos métodos de amostragem quantitativa quando se utilizam redes. Um desses problemas está relacionado com a fuga de pequenos organismos 
através das malhagens. De acordo com Ahlstrom (1973) é impraticável o uso de malhagens menores que $0,333 \mathrm{~mm}$ nas coletas porque favorece 0 entupimento e conseqüente redução da água filtrada, que aumenta consideravelmente abaixo dessa abertura. Para Saville (1957) a fuga de organismos é devido, em parte, a distorção da malha provocada pela compressão dos organismos contra a parede da rede.

Nichols \& Thompson (1991), estudando a influência da abertura de malha na coleta de 4 espécies dos copépodos Calanus spp, Pseudocalanus minutus, Paracalanus parvus e Temora longicornis concluíram que, para que $95 \%$ dos indivíduos presentes na água do mar amostrada possam ser capturados, é necessário que a abertura da malha seja equivalente a pelo menos $75 \%$ da largura dos copépodos. $O$ aparelho de coleta utilizado no presente trabalho foi a rede Bongô com abertura de malha de $0,333 \mathrm{~mm}$, que teria atuado como elemento selecionador dos organismos capturados, favorecendo a fuga principalmente dos estágios de copepódito IIII. Assim, a rede Bongô mostrou ser um aparelho inconveniente para amostragens quantitativas de organismos de pequeno tamanho como $P$. quasimodo, $P$. indicus e $P$. aculeatus que, segundo Björnberg (1981), possuem comprimentos de 0,75$0,82 \mathrm{~mm} ; 0,75-0,8 \mathrm{~mm}$ e $0,92-1,2 \mathrm{~mm}$, respectivamente.

A biomassa zooplanctônica, na zona eufótica, tende a ser maior à noite que durante o dia (Napp et al., 1988; Hays, 1995). Migradores verticais como os copépodos Calanoida, deslocam-se para a superficie ao anoitecer e descem para as camadas mais profundas ao amanhecer (Pagano et al., 1993), evitando sua exposição aos predadores visuais (Zaret \& Kerfoot, 1975; Chae \& Nishida, 1995; Hays et al., 1995). A migração pode ser provocada também pela intolerância aos raios ultravioletas (Johnson, 1946 apud Vinogradov, 1970), os baixos teores de oxigênio da água (Sameoto, 1986), a temperatura e a concentração de matéria orgânica particulada (Paffenhöfer, 1983).

Brooks \& Mullin (1983) verificaram que os pequenos herbívoros permanecem próximo à superfície na zona eufótica, onde o fitoplâncton é abundante. Björnberg \& Wilbur (1968), correlacionaram a intensidade de migração vertical (MV) com a forma de alimentação: MV bem marcada em organismos predadores (Calanopia americana); MV praticamente ausente em filtradores (Paracalanus crassirostris) e MV de intensidade intermediária para os omnívoros (Acartia lilljeborgi). Em contraposição, os copépodos de regiões neríticas que normalmente exibem migração vertical diária, não migram quando expostos a uma concentração de alimento elevada, permanecendo nas camadas superficiais durante todo o dia (Fransz et al.,1984; Dagg, 1985).

Tang et al. (1994) relataram que as espécies de Paracalanus permanecem em águas profundas durante o dia, para evitar a pressão de predação, e migram para a superficie à noite a fim de se alimentar. Para Ohman et al. (1983), os Paracalanus não migram ou realizam migração inversa, isto é, deslocam-se para a superficie durante o dia, na presença de grande quantidade de predadores.

Na região de São Sebastião, as 3 espécies de Paracalanus estudadas ocorreram em maior número acima da termoclina, durante o período da noite. Essa distribuição é típica de organismos que realizam migração vertical com a finalidade de alimentar-se e/ou de fugir dos predadores visuais. Ao que tudo indica, a forte estratificação termohalina na região durante $o$ período de amostragens, não foi uma barreira térmica para que os Paracalanidae pudessem realizar a migração vertical. A presença de matéria orgânica seria um outro fator que pode ter influenciado a ocorrência e distribuição de $P$. quasimodo, $P$. indicus e $P$. aculeatus. Segundo Gianesella-Galvão et al. (1996), a existência da ilha de São Sebastião, com a presença de matas exuberantes até quase a linha d'água, permite que a matéria orgânica de origem vegetal seja carregada pelo "run-off" e chegue pouco decomposta ao ambiente marinho. Ao sofrer decomposição, libera amônia, tornando essa massa de água mais produtiva. Isto justificaria, em parte, os valores de densidade relativamente altos observados durante $o$ período de coleta ( 24 horas).

\section{Agradecimentos}

Os autores agradecem à Profa. Dra. Thaïs Navajas Corbisier e a M.Sc. Emília Arasaki pelo auxílio na análise de agrupamentos. À M.Sc. Liang Tsui Hua e aos técnicos do IOUSP pelo auxílio nos trabalhos de campo. Aos dois Assessores anônimos, pelas críticas e sugestões feitas aos manuscritos. À Fundação de Amparo à Pesquisa do Estado de São Paulo - FAPESP (Proc. $\mathrm{n}^{\circ}$ 92.3449/0) pelo apoio financeiro dado ao presente trabalho. Ao $\mathrm{CNPq}$ (Programa PIBIC/USP) e à CAPES pelas bolsas de estudo fornecidas. 


\section{Referências bibliográficas}

Aidar, E.; Gaeta, S. A.; Gianesella-Galvão, S. M. F.; Kutner, M. B. B \& Teixeira, C. 1993. Ecossistema costeiro subtropical: nutrientes dissolvidos, fitoplâncton e clorofila- $a$ e suas relações com as condições oceanográficas na região de Ubatuba, SP. Publção esp. Inst. oceanogr., S Paulo, (10):9-43.

Ahlstrom, E. H. 1973 Ichthyoplankton surveys for detection and appraisal of fishery resources. FAO Fish. tech. Pap., 122:3-13.

Angel, M. V. 1984. Detrital organic fluxes through pelagic ecossystems. In: Fasham, M. J. R., ed. Flows of energy and materials in marine ecosystems: theory and pratice. New York, Plenum Press. p. 475-516.

Björnberg, T. K. S. 1963. On the marine free-living copepods off Brazil. Bolm Inst. oceanogr. S Paulo, 13(1):3-142.

Björnberg, T. K. S. 1980. Revisão da distribuição dos gêneros Paracalanus, Clausacalanus e Ctenocalanus (Copepoda, Crustacea) ao largo do Brasil. Bolm. Inst. oceanogr., S Paulo, 29(2):6568.

Björnberg, T. K. S. 1981. Copepoda. In: Boltovskoy, D. ed. Atlas de zooplancton del Atlántico Sudoccidental y métodos de trabajo con zooplancton marino. Mar del Plata, Instituto Nacional de Investigación y Desarrollo Pesquero. p.587-679.

Björnberg, T. K. S. \& Wilbur, K. M. 1968. Copepod phototaxis and vertical migration influenced by xanthene dyes. Biol. Bull. mar. biol. Lab., Woods Hole, 134(3):389-410.

Bowman, T. E. 1971. The distribution of calanoid copepods off the Southeastern United States between Cape Hatteras and Southern Florida. Smithsonian Contr. Zool., 96:1-58.

Brooks, E. R. \& Mullin, M. M. 1983. Diel changes in the vertical distribution of biomass and species in the southern California Bight. CALCOFI Rep., $24: 210-215$
Chae, J. C. \& Nishida, S. 1995. Vertical distribution and diel migration in the iridescent copepods of the Family Sapphirinidae: a unique example of reverse migration? Mar. Ecol. Prog. Ser., 119:111-124.

Coyle, K. O.; Paul, A. J. \& Ziemann, D. A. 1990. Copepod populations during the spring bloom in an Alaskan Subarctic embayment. J. Plankt. Res., 12(4):759-797.

Dagg, M. G., 1985. The effects of food limitation on diel migratory behavior in marine zooplankton. Arch. Hydrobiol. Beih., 21:247-255.

Damkaer, D. M. 1996. Copepod taxonomy: Discovery vs. recognition. Proc. Biol. Soc. Wash., 109(4):687-694.

Eisma, D. \& Kalf, J. 1987. Distribution, organic content and particle size of suspended matter in the North Sea. Neth. J. Sea. Res., 21:265-285.

Fransz, H. G.; Miquel, J. C.; Gonzalles, S. R. 1984. Mesozooplancton composition, biomass and vertical distribution and copepod production in the stratified Central North Sea. Neth. J. Sea Res., 18(1/2):82-96.

Gaeta, S. A.; Abe, D. S.; Susini, S. N.; Lopes, R. M.; Metzler, P. M. 1990. Produção primária, plâncton e covariáveis ambientais no Canal de São Sebastião, durante o outono. Rev. Brasil. Biol., 50(4):963-974.

Gianesella-Galvão, S. M. F.; Aidar, E.; Vega-Pérez, L. A. \& Saldanha-Corrêa, F. M. P. 1996. Estudo do plâncton e produção primária da plataforma interna da região de São Sebastião. In: Oceanografia da Plataforma Interna da Região de São Sebastião. Relatório FAPESP, (3):70-163.

Gonzáles, H. E.; Kurbjeweit, F. \& Bathmann, U. V. 1994. Occurrence of cyclopoid copepod and faecal material in the Halley Bay region, Antarctica, during January-February 1991. Polar Biol., 14(5):331-342.

Grant, G. C. 1991. Chaetognatha from the central and southern middle Atlantic Bight. Species composition, temperature-salinity relationships, and interspecific associations. Fish. Bull. U.S., 89:33-40. 
Hays, G. C. 1995. Ontogenetic and seasonal variation in the diel vertical migration of the copepods Metridia lucens and Metridia longa. Limnol. Oceanogr., 40(8):1461-1465.

Hays, G. C.; Warner, A. J. \& Proctor, C. A. 1995. Spatio-temporal patterns in the diel vertical migration of the copepod Metridia lucens in the northeast Atlantic derived from the Continuous Plankton Recorder survey. Limnol. Oceanogr., 40(3):469-475.

Hillgruber, N; Haldorson, L. J. \& Paul, A. J. 1995. Feeding selectivity of larvae walleye pollock Theragra chalcogramma in the oceanic domain of the Bering Sea. Mar. Ecol. Prog. Ser., 120:110.

Horn, H. S. 1966. Measurement of "overlap" in comparative ecological studies. Am. Nat., 100:419-424.

Hopcroft, R. R. \& Roff, J. C. 1996. Zooplankton growth rates: diel egg production in the copepods Oithona, Euterpina and Corycaeus from tropical waters. J. Plankt. Res., 18(5):789-803.

Katsuragawa, M. 1985. Estudo sobre a variabilidade de amostragem, distribuição e abundância de larvas de peixes da região sudeste do Brasil. Dissertação de mestrado. Universidade de São Paulo, Instituto Oceanográfico. 168p.

Katsuragawa, M. 1995. Estudo do ictioplâncton da plataforma interna da região de São Sebastião. In: Oceanografia da Plataforma Interna da Região de São Sebastião. Relatório FAPESP, (2):164-206.

Kiørboe, T.; Kaas, H.; Krause, B.; Møhlenberg, F.; Tiselius, P. \& Ærtebjerg, G. 1990. The structure of the pelagic food web in relation to water column structure in the Skagerrak. Mar. Ecol. Prog. Ser., 59:19-32.

Kouwenberg, J. H. M. 1994. Copepod distribution in relation to seasonal hydrographics and spatial structure in the north-western Mediterranean (Golfe du Lion). Est. Coast. Shelf. Sci., 38(1):69. 90.

Kramer, D.; Kalin, M. J.; Stevens, E. G.; Treikill, J. R. \& Zweifel, J. R. 1972. Collecting and processing data on fish eggs and larvae in the California Current region. U.S. NOAA tech. Rept, (370):1-38.
Lawson, T. J. \& Grice, G. D. 1973. The developmental stages of Paracalanus crassirostris Dahl, 1894 (Copepoda, Calanoida). Crustaceana, 24:43-56.

Legendre, L. \& Legendre, P. 1983. Numerical ecology. Amsterdam, Elsevier. 419 p.

Mann, K. H. 1991. Organisms and ecosystems. In: Barnes, R.S. \& Mann, K. H. eds. Fundaments of aquatic ecology, $2^{\text {gd }}$ ed. London, Blackwell Scientifi Publications, p. 3-28.

Nakata, K. 1988. Alimentary tract contents and feeding conditions of ocean-caught post larval japanese sardine, Sardinops melanostictus. Bull. Tokai reg. Fịh. Res, Lab., 126:11-24.

Napp, J. M.; Brooks, E. R.; Matrai, P. \& Mullin, M. M. 1988. Vertlegl distribution of marine particles and grazers. II. Relation of grazer distribution to food quality and quantity. Mar. Ecol. Prog. Ser., 50:59-72.

Nichols, J. H. Thompson, A. B. 1991. Mesh selection of eopepodite and nauplius stages of four calanoid eopepod species. J. Plankt. Res., 13(3):661-671.

Nielsen, T. G. \& Sabatini, M. 1996. Role of cyclopoid copepods Oithona spp. in North Sea plankton communities. Mar. Ecol. Prog. Ser., 139:79-93.

Ohman, M. D., Frost, B. W. \& Cohen, E. B. 1983. Reverse diel vertical migration: an escape from invertebrate predators. Science, 220:1404-1407.

Omori, M. \& Ikeda, T. 1992. Methods in marine zooplankton ecology. $2^{\text {nd }}$ ed. New York, John Wiley. $332 \mathrm{p}$.

Paffenhöfer, G.-A. 1983. Vertical zooplankton and distribution on the northeastern Florida shelf and its relation to temperature and food abundance. $J$. Plankt. Res., 5(1):15-34.

Paffenhöfer, G.-A. \& Knowles, S. C. 1979. Ecological implications of fecal pellet size, production and consumption by copepods. J. Mar. Res., 37:35-49. 
Pagano, M.; Gaudy, R.; Thibault, D. \& Lochet, F. 1993. Vertical migration and feeding rhythms of mesozooplanktonic organisms in the Rhône River Plume Area (North-west Mediterranean Sea). Estuar. coast. Shelf. Sci., 37:251-269.

Pedreira, M. M. 1997. Alimentação e hábito alimentar de larvas de Trachurus lathani (Família Carangidae), na região de Ubatuba, Estado de São Paulo. Dissertação de mestrado. Universidade de São Paulo, Instituto Oceanográfico.129p.

Raymont, T. E. 1983. Plankton and productivity in the oceans. Zooplankton. Oxford, Pergamon Press. v.2.

Razouls, S \& Thiriot, A. 1968. Le macrozooplankton de la région de Banyuls-sur-Mer (Golfe du Lion). Vie Millieu, 19.(1B):133-184.

Richerson, P. J.; Powell, T. M.; Leigh-Abbot, M. R. \& Coil, J. A. 1978. Spatial heterogeneity in closed basins. In: Steele, J. H. ed. Spatial pattern in plankton communities. London, Plenum Press. p.239-276.

Rippingale, R. J. 1994. A calanoid copepod Gladioferens imparipes, holding to surface. Hydrobiologia, 292/293:351-360.

Saiz, E. \& Alcaraz, M. 1991. Effects of small-scale turbulence on development time and growth of Acartia grani (Copepoda: Calanoida). J. Plankt. Res., 13(4):873-883.

Sameoto, D. D. 1986. Influence of the biological and physical environment on the vertical distribution of mesozooplankton and micronekton in the eastern tropical Pacific. Mar. Biol., 93(2):263279.

Sasaki, H.; Hattori, H.\& Nishizawa, S. 1988. Donward flux of particulate organic matter and vertical distribution of calanoid copepods in the Oyashio water in Summer. Deep-Sea Res., 35(4):505-515.

Saville, A. 1957. Mesh selection in plankton nets. J. Conseil, 23(1):192-201.

Seguin, G.; Errhif, A. \& Dallot, S. 1994. Diversity and structure of pelagic copepod populations in the frontal zone of the eastern Alboran sea. Hydrobiologia, 292/293:369-377.
Smetacek, V. S. 1980. Zooplankton standing stock, copepod faecal pellets and particulate detritus in Kiel Bight. Estuar. coast. mar. Sci., 2:477-490.

Soetaert, K. \& Herman, P. M. 1994. One foot in the grave: Zooplankton drift into the Westerschelde estuary (The Netherlands). Mar. Ecol. Prog. Ser., 105:19-29.

Tang, K. W.; Chen, Q. C.; Wong, C. K. 1994. Diel vertical migration and gut pigment rhythm of Paracalanus parvus, P. crassirostris, Acartia erythraea and Calanus subcrassus (Copepoda, Calanoida) in Tolo harbour, Hong Kong. Hybrobiologia, 292/293:389-396.

Turner, J. T. 1994. Planktonic copepods of Boston Harbor, Massachusetts Bay and Cape Cod Bay, 1992. Hydrobiologia, 292/293:405-413.

Valdes, J. L.; Roman, M. R.; Alvarez-Ossorio; M. T., Gauzens; A. L. \& Miranda, A. 1990. Zooplankton composition and distribution off the coast of Galicia, Spain. J. Plankt. Res., 12(3):629643

Vega-Pérez, L. A. 1993. Estudo do zooplâncton da região de Ubatuba, Estado de São Paulo. Publção esp. Inst. oceanogr., S Paulo, (10):65-84.

Vinogradov, M. E. 1970. Vertical distribution of the zooplankton. Israel Program for Scientific Translation. 339p.

Zaret, T. M. \& Kerfoot, W. C. 1975. Fish predation on Bosmina longirostris. Body-size selection versus visibility selection. Ecology, 56:232-237.

(Manuscrito recebido 20 fevereiro 1997; revisado 28 abril 1997; a aceito 26 junho 1997) 